\title{
Broadband mid-IR on-chip Fourier-transform Spectrometer
}

\author{
Miguel Montesinos-Ballester, ${ }^{1, *}$, Qiankun Liu ${ }^{1}$, Vladyslav Vakarin ${ }^{1,3}$, Joan Manel Ramirez ${ }^{1,4}$, Carlos Alonso-Ramos ${ }^{1}$, \\ Xavier Le Roux ${ }^{1}$, Jacopo Frigerio ${ }^{2}$, Andrea Ballabio ${ }^{2}$, Enrico Talamas ${ }^{2}$, Laurent Vivien ${ }^{1}$, Giovanni Isella ${ }^{2}$, and Delphine \\ Marris-Morini ${ }^{1}$ \\ ${ }^{1}$ Centre de Nanosciences et de Nanotechnologies, CNRS, Univ. Paris-Sud, Université Paris-Saclay, 91120 Palaiseau, France \\ ${ }^{2}$ L-NESS, Dipartimento di Fisica, Politecnico di Milano, Polo di Como, Via Anzani 42, 22100 Como, Italy \\ ${ }^{3}$ Now in IMEC, Kaperldreef 75, 3001 Leuven, Belgium \\ ${ }^{4}$ Now in III-V Lab, 91120 Palaiseau, France \\ *e-mail: miguel.montesinos@u-psud.fr
}

\begin{abstract}
We present a new FTS approach that harnesses both spatial heterodyning and thermo-optical path tuning to overcome the resolution-bandwidth tradeoff in conventional counterparts. We experimentally demonstrate a mid-infrared SiGe FTS, with a resolution exceeding 15 $\mathrm{cm}^{-1}$ in a wide bandwidth of $603 \mathrm{~cm}^{-1}$.
\end{abstract}

Keywords - Silicon photonics, Spectroscopy, mid-infrared

\section{INTRODUCTION}

The mid-IR spectral range, and more specifically the wavelength region between 5 and $20 \mu \mathrm{m}$, aroused a great interest in the Photonic Integration Circuit (PIC) community due to its great potential in spectroscopy and sensing $[1,2]$. That is the reason why the development of photonic platforms dedicated to long MIR wavelengths has recently witnessed a burst of research activity [3]. Germanium-rich silicon-germanium (Ge-rich $\mathrm{SiGe}$ ) has emerged as a promising integrated platform exhibiting a wide transparency range [4]. A linearly graded SiGe layer allows a smooth transition between pure silicon and Ge-rich material that minimizes the threading dislocation density due to lattice mismatch, while confining the optical mode in the upper part of the waveguide by refractive index gradient. The recent experimental demonstration of a MIR spectrometer in this platform [5] provides a promising departing point to develop new deep MIR spectroscopic systems. All these compelling features make the Ge-rich $\mathrm{SiGe}$ an excellent platform for developing mid-IR spectroscopy PICs.

In the last years, a myriad of integrated spectroscopic systems has been demonstrated [6-8]. Among them, on-chip Fourier-transform spectrometers (FTS) implement simple yet effective calibration algorithms that compensate phase and amplitude impairments, thereby providing a superior robustness against fabrication imperfections compared to dispersive counterparts. Within FTS, on-chip Spatial Heterodyne Spectroscopy (SHS) approach is based on an array of interferometers with linearly increasing optical path delay to achieve a remarkably resolution without any moving part. However, its operational bandwidth is limited by the minimum optical path difference between consecutive interferometers. The thermo-optic effect has also been used to scan the optical path delay of a single interferometer [9], but the resolution of these devices is limited by the maximum achievable optical delay.
In this work we propose and experimentally demonstrate a new FTS approach that gathers the advantages of spatial heterodyning and optical path tuning by thermo-optic effect to overcome the bandwidth-resolution tradeoff in conventional counterparts. This device is fabricated in a graded-index SiGe platform which has been demonstrated to have low propagation losses up to $8.5 \mu \mathrm{m}$ wavelength [4].

\section{WORKING PRINCIPLE AND EXAMPLE}

The operation principle of integrated spatial heterodyne Fourier-transform spectrometers (SHFTS) has been previously reported in [10]. We propose here to achieve a broadband and high-resolution mid-IR spectrometer, by generating different path delays by both arm length difference and thermal scan sampling. For this purpose, the mathematical model of the MZI array is generalized to thermally-tuned MZI array. Then, the resolution and Free Spectral Range (FSR) of the thermally-tuned SHFTS are given by:

$$
\begin{gathered}
\delta \sigma=\frac{1}{\gamma_{\max }}=\frac{1}{\Delta L_{\max } n_{g}+L_{H} \Delta n_{g, T \max }} \\
F S R=\frac{1}{\Delta \mathrm{L}_{\max } \mathrm{n}_{\mathrm{g}}+L_{H} \Delta n_{g, T \max }} \frac{N_{T} N_{L}}{2}
\end{gathered}
$$

where $\gamma_{\max }$ is the optical path delay difference, $\Delta n_{g, T \max }$ is the maximum group index change due to thermal difference, $N_{T}$ is the different number of applied temperatures and $N_{L}$ is the number of MZIs in the array.

To demonstrate the benefits and viability of the thermallytuned FTS, we consider an array of 10 MZIs (9 unbalanced and 1 balanced MZI), with a path difference increase $\left(\Delta L_{\text {step }}\right)$ of $18.8 \mu \mathrm{m}$ between them. A $3 \mathrm{~mm}$-long heater is located in the shortest arm of each MZI, too. As the optical mode is confined in Ge-rich SiGe materials, the thermo-optic coefficient of $\mathrm{Ge}$ is used and is also considered constant within the operational wavelength range with a value of $4.1 \times 10^{-4} \mathrm{~K}^{-1}[11]$.

In Fig. 1 is shown the simulated retrieval process of a monochromatic input (black arrow) in a $551 \mathrm{~cm}^{-1}$ bandwidth for different cases. The retrieved spectrum shown in Fig. 1(a) illustrate the classical SHS-based FTS without any thermal tuning. In Fig. 1(b) and 1(c) the thermal tuning is implemented using a uniform sampling of the optical path delay for 4 and 9 different applied temperatures, with a maximum thermal 
increase of $54 \mathrm{~K}$. In this figure is clearly seen the FSR enhancement when increasing the number of applied temperatures, and then reducing the minimum path delay difference between samples, without affecting the resolution performance.
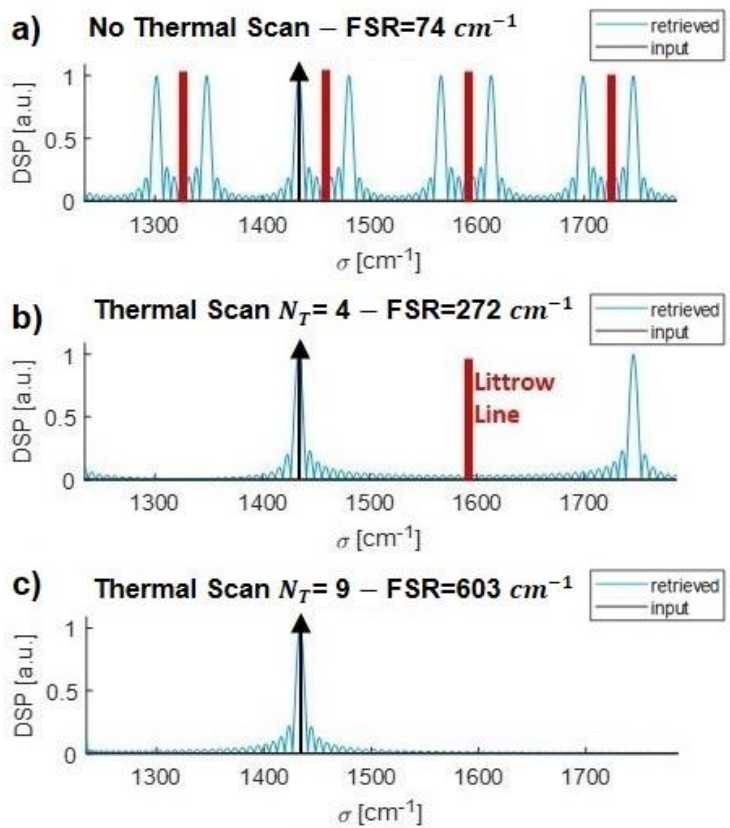

Fig. 1. Retrieval process of a monochromatic input centered at $1434 \mathrm{~cm}^{-1}$ for different cases. (a) Non thermal tuning. (b) 4 differenet applied temperatures with linear path delay sampling. (c) 9 different applied temperatures with linear path delay sampling. Littrow lines are reported in red.

\section{EXPERIMENTAL DEMONSTRATION}
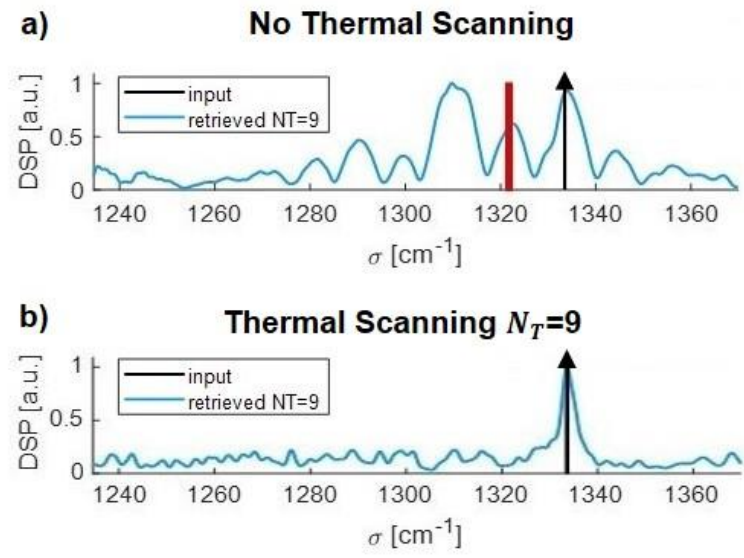

Fig. 2. Experimental retrieval process in a wavelength range from 7.3 to 8.1 $\mu \mathrm{m}$. (a) Retrieved spectrum with non-thermal tuning. (b) Retrieved spectrum with $N_{T}=9$ and a maximum applied temeprature increase of $54 \mathrm{~K}$.

The thermally-tuned FTS has been evaluated experimentally using a $10 \mathrm{MZI}$ array with an $\Delta L_{s t e p}$ of $18.8 \mu \mathrm{m}$. The theoretical resolution and FSR of such array without any thermal-tuning are $14.6 \mathrm{~cm}^{-1}$ and $74 \mathrm{~cm}^{-1}$ respectively. Heaters have been added in the shortest arm of each MZI. A maximum temperature variation of $3 K$ has been applied on each MZI, which is much smaller than $\Delta T_{\max }=54 \mathrm{~K}$ to have a uniform sampling of the optical path delay. Interestingly, the thermally-tuned FTS is also working in this case, where the gamma sampling is not uniform. Indeed, we can see in Fig. 2(a) that a FSR of $74 \mathrm{~cm}^{-1}$ is not enough to properly retrieve the input a monochromatic signal in a $132 \mathrm{~cm}^{-1}$ bandwidth range. In comparison, the use of the thermal tuning allows to increase the FSR up to $603 \mathrm{~cm}^{-1}$, which is much larger than the measurement window and thus a good signal retrieval is obtained.

\section{CONCLUSION}

This work shows that it is possible to harness the thermooptic effect to enhance the performance of on-chip FTS. This effect can be used to increase the FTS performance, to reduce the number of MZI in the array to achieve given specifications, thus reducing the device footprint, or even both at the same time. These results provide to the best of our knowledge, the first experimental demonstration of on-chip MIR Ge-based FTS exploiting both spatial heterodyning and temperature tuning. On-chip integration of sensing circuits with the FT spectrometer could pave the way for future demonstration of robust, highresolution, and cost-effective multi-target spectrometers covering an ultrawideband of the fingerprint wavelength range.

\section{ACKNOWLEDGMENTS}

This work was supported by the European Research Council (ERC) under the European's Union Horizon 2020 ( No 639107 INsPIRE). The fabrication of the device has been performed in the Plataforme de Micro-Nano-Technologie/C2N, which is partially funded by the "Conseil Géneral de l'Essone". This work was partly supported by the French RENATECH network.

\section{REFERENCES}

[1] A. Mihcel et al., "Long-Path Quantum Cascade Laser-Based Sensor for Methane Measurements", J. Atmos, Ocean Technol., vol. 33, pp. 23732384, Nov. 2016

[2] G. Reich, "Near-infrared spectroscopy and imaging: Basic principles and pharmaceutical applications". Adv. Drug Deliv. Rev, vol. 57, pp. 11091143, June 2005.

[3] G. Roelkens et al., "Mid-IR heterogeneous silicon photonics", Proc. SPIE vol. 8993, p. 899316, Jan. 2014.

[4] J. M. Ramirez et al., "Graded SiGe waveguides with broadband low-loss propagation in the mid infrared", Opt. Express, vol. 26, pp. 870-877, Jan. 2018.

[5] Q. Liu et al., "Integrated broadband dual-polarization Ge-rich SiGe midinfrared Fourier-transform spectrometer", Opt. Lett., vol. 43, pp. 50215024, 2018.

[6] E. Le Coarer et al., "Wavelength-scale stationary-wave integrated Fourier-transform spectrometry", Nat. Photonics, vol. 1, pp. 473-478, Aug. 2007.

[7] D. Kita et al., "High-performance and scalable on-chip digital Fourier transform spectroscopy", Nat. Commun., vol. 9, pp. 4405, Oct. 2018.

[8] R. A. Soref, F. De Leonardis,V. M. Passaro and Y. Fainman, "On-Chip Digital Fourier-Transform Spectrometer Using a Thermo-Optical Michelson Grating Interferometer", J. Light. Technol., vol. 36, pp. 51605167, Aug. 2018.

[9] M. C. Souza, A. Grieco, N. C. Frateschi and Y. Fainman, "Fourier transform spectrometer on silicon with thermo-optic non-linearity and dispersion correction", Nat. Commun., vol. 9, p. 665, Feb. 2018.

[10] M. Florjanczyk et al., "Multiaperture planar waveguide spectrometer formed by arrayed Mach-Zehnder interferometers", Opt. Express, vol. 15, pp. 18176-18189, 2007.

[11] B. J. Frey, D. B. Leviton and T. J. Madison, "Temperature-dependent refractive index of silicon and germanium", Proc. of SPIE (Orlando U.S.), vol. 6273, p. 62732J, 2006. 